How to cite this article:

Hekmatzadeh AA, Torabi Haghighi A,Hosseini Guyomi K, Amiri SM, Kløve B. The effects ofextremes and temporal scale on multifractal properties of riverflow time series.River Res Applic. 2019;1-12.https://doi.org/10.1002/rra.3550

\title{
The effects of extremes and temporal scale on multifractal properties of river flow time series
}

Ali Akbar Hekmatzadeh ${ }^{\mathrm{a}}$, Ali Torabi Haghighi ${ }^{\mathrm{b} *}$, Kianoosh Hosseini Guyomi $^{\mathrm{a}}$, Seyed Mehrab Amiri ${ }^{\mathrm{c}}$, Bjørn Kløve $^{\mathrm{b}}$

${ }^{a}$ Department of Civil and Environmental Engineering, Shiraz University of Technology, Shiraz, Iran.

${ }^{\mathrm{b}}$ Water, Energy and Environmental Engineering unit, University of Oulu, Finland.

${ }^{\mathrm{C}}$ Department of Civil and Environmental Engineering, Shiraz University, Shiraz, Iran.

*Corresponding author: Ali Torabi Haghighi, email: ali.torabihaghighi@oulu.fi

Running head: Multifractality of river flow 


\title{
The effects of extremes and temporal scale on multifractal properties of river flow time series
}

\begin{abstract}
For accurate forecasting of extreme events in rivers, streamflow time series with sub-daily temporal resolution (1-6 hour) are preferable, but discharge time series for long rivers are usually available at daily or monthly resolution. In this study, the scaling properties of hourly and daily streamflow time series were measured. As an innovation, the effects of extreme values on the multifractal behavior of these series were evaluated. Interestingly, both hourly and daily discharge records led to nearly identical scaling trends and identical crossover times. Daily and hourly discharge time series appeared to be non-stationary when the timescale ranged from 75 to 366 days. Otherwise, the signals may be considered stationary time series. In addition, the results indicated that the extreme values strongly contribute to the multifractality of the series. The width of singularity spectra decreased considerably when the extreme events were removed from both hourly and daily discharge records.
\end{abstract}

Keywords: MF-DFA, extreme events, hourly discharge time series, daily discharge time series, fractal 


\section{Introduction}

During the past 40 years, it has been acknowledged that climatological and hydrological parameters such as precipitation and river flow rate follow scaling properties or power-law correlations on different timescales (Koscielny-Bunde, Kantelhardt, Braun, Bunde, \& Havlin, 2006; Rehman, 2009). If the behavior of a system obeys the scaling law, it is termed a fractal system. In such a case, self-similarity is defined where the system's general behavior is reiterated at arbitrarily smaller and smaller scales (Breslin \& Belward, 1999). Mathematically, if $x$ is a function of $t$ and $x(\alpha t)$ is similar to $x(t)$ with a scaling factor of $\alpha$, the system shows self-similar behavior. Regarding time series, the term "self-affinity" is more applicable, where in a self-affine fractal system, $\alpha^{H} x(\alpha t)$ is similar to $\mathrm{x}(\mathrm{t})$. Here, the exponent $\mathrm{H}$ is called the Hurst exponent. In addition, a dataset can be characterized as monofractal when a single scaling exponent is adequate to describe its fractal features, whereas multifractality stems from the presence of numerous scaling exponent (Kantelhardt, 2009).

fractal analysis can be used as a statistical technique to identify measures of weak and strong persistence in self-affine time series (Malamud \& Turcotte, 1999), variations of time scales in datasets (Kuai \& Tsai, 2012), and patterns of temporal scaling (X. Yu, Ghasemizadeh, Padilla, Kaeli, \& Alshawabkeh, 2016). It is also applicable in the detection of long-range correlations, which can be considered as a powerlaw decay of the correlation function (Al Sawaf, Kawanisi, Kagami, Bahreinimotlagh, \& Danial, 2017). These scaling behaviors may improve the accuracy of statistical methods to predict rare events in river flow time series.

Detrended Fluctuation Analysis (DFA), developed by Peng et al. (1994)), is a useful method for examining the fractal properties of time series, as it can deal with both stationary and non-stationary signals. This method has been widely employed to determine long-range correlations in different fields of science 
associated with time series, e.g., meteorological studies (Rehman, 2009), disease diagnosis (Dutta, Ghosh, \& Chatterjee, 2018), physics (Saha, Shaw, Ghosh, Janaki, \& Sekar Iyengar, 2018), and financial and stock markets (Rizvi \& Arshad, 2016).

The DFA approach reveals monofractal properties of natural records, but many hydrological processes need several scaling exponents to be described, while simple DFA is not capable of detecting multifractal characteristics of time series (Kalamaras, Philippopoulos, Deligiorgi, Tzanis, \& Karvounis, 2017). Accordingly, Kantelhardt et al. (2002) developed Multifractal Detrended Fluctuation Analysis (MFDFA), which eliminates the shortcomings of DFA. A number of natural time series have been successfully described recently using the MF-DFA, including air temperature (Kalamaras et al., 2017), sediment load (Kuai \& Tsai, 2012), rainfall (Krzyszczak, Baranowski, Zubik, \& Hoffmann, 2017), wind speed (Cadenas et al., 2019), groundwater level fluctuations (Habib et al., 2017), sea level oscillations (Y. Zhang \& Ge, 2013), earthquake acceleration (Fan \& Lin, 2017), heart rate (Piskorski et al., 2018), and financial time series (Teng \& Shang, 2018).

For river flow, the daily discharge fluctuations in several large rivers have been analyzed using DFA (Livina et al., 2003). However, Koscielny-Bunde et al. (2006)) observed multifractal properties in streamflow fluctuations at several hydrological river stations. Movahed \& Hermanis, (2008)) detected multifractal correlation in monthly discharge records of the Daugava river in Latvia. Similar fractal behavior has been reported for the Yangtze river in China (Q. Zhang, Zhou, Singh, \& Chen, 2011). Water level fluctuations in Brazilian rivers have been investigated by Rego, Frota, \& Gusmão (2013), whose results indicated the existence of multifractality. Al Sawaf et al. (2017) tried to determine the scaling properties of a mountainous river in Japan using DFA.

The majority of fractal studies on streamflow time series were based on original discharge data. However, no attention has been paid to consider the effects of large floods or extreme events on the fractal properties of river discharge data. The research about extreme events usually relies on classical extreme 
value theory or different methods of frequency analysis (Hernández Ayala, Keellings, Waylen, \& Matyas, 2017; Salas, Obeysekera, \& Vogel, 2018). The extreme events in rivers require more studies using different statistical tools, as flooding can cause billions of dollars' worth of damage (Tawn, Shooter, Towe, \& Lamb, 2018).

In addition, Historical records of river discharge data are generally available only as daily or monthly data, especially in developing countries (Ashraf et al., 2016, Torabi Haghighi, Marttila \& Klöve, 2014, Rebora, Silvestro, Rudari, Herold, \& Ferraris, 2016; Reynolds, Halldin, Xu, Seibert, \& Kauffeldt, 2017). Nonetheless, sub-daily streamflow datasets are required for many hydrological applications, such as prediction of extreme values in rivers or hydropeaking analysis (Ashraf et al., 2018, Torabi Haghighi et al. , 2019). In the case of small or medium-sized basins, where time concentrations are smaller than 24 hours, daily time series are not representative of flood events, since the instantaneous peak flow is greater than mean daily flow (Maskey, Puente, \& Sivakumar, 2019; Reynolds et al., 2017; Ronchail et al., 2018). This indicates the significance of temporal scale problems in hydrological prediction models (Hoffmann et al., 2017; Krzyszczak et al., 2017). Therefore, it is essential to measure and compare the characteristics of discharge time series with fine and coarse time resolution (e.g. hourly and daily discharge data).

This study examined the effect of time aggregation or time downscaling on the fractal properties of streamflow time series in an arid/semi-arid region. In a novel approach, extreme values were removed from the discharge time series in order to detect variations in the fractal properties of the new time series. As an empirical case, hourly and daily discharge time series of the Shapur river in southern Iran were analyzed using DFA and MF-DFA. Datasets from two gauging stations on the river with the longest records were selected.

\section{Material and Methods}

\subsection{Study area and data}


The Shapur river originates northeast of Kazeroon, a city in the west of Fars Province, Iran (Fig. 1). The river is $220 \mathrm{~km}$ long, has a drainage area of $4500 \mathrm{~km}^{2}$, and has annual discharge of approximately 11 $\mathrm{m}^{3} \mathrm{~s}^{-1}$ (Afshin, 1994). The Shapur river joins the Dalaki river in Bushehr province to form the Helleh river, which discharges into the Persian Gulf. Moreover, several cultural and historical sites of the ancient Sasanian empire are located along the river banks, increasing the significance of accurate flood prediction. Mean annual precipitation in the Kazeroon region is $341 \mathrm{~mm}$ and maximum temperature from July to September ranges between 39 and $41^{\circ} \mathrm{C}$, indicating a tropical climate (Iran Meteorological Organization, 2018).

For this study, hourly and daily discharge time series from the Bushigan gauging station (2943'6”N;

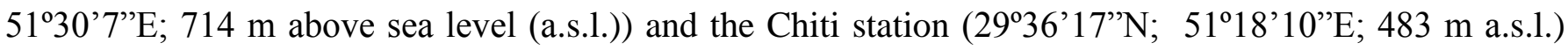
were obtained from Fars Regional Water Authority (Fig. 1). There are also two other gaging stations on this river with shorter series of data records. For Bushigan and Chiti stations, daily discharge data are available from 1978, while hourly discharge data are available from 2005 (see Fig. 2). The daily flow rate at Chiti and Bushigan gauging stations shows significant variations (range 1-923 and 0.3-467 $\mathrm{m}^{3} \mathrm{~s}^{-1}$, respectively).

\subsection{Power Spectrum (PS)}

The Power Spectrum is a conventional statistical tool for detecting self-affinity in hydrological time series (Rakhshandehroo \& Amiri, 2012). If the spectrum (E(f)) follows the power-law formula (Eq. 1), the data exhibit scaling property or fractal behavior.

$$
\mathrm{E}(\mathrm{f})=\mathrm{cf}^{-\beta}
$$

where $f$ is frequency and $\beta$ is the spectral exponent.

\subsection{Detrended and Multifractal Detrended Fluctuation Analysis (DFA and MF-DFA)}


The DFA method is widely employed to extract the Hurst exponent from time series (C.-K. Peng et al., 1994; C. K. Peng, Havlin, Stanley, \& Goldberger, 1995). At first, a cumulative time series (y(i)) is derived from the original series using Eq. 2. measuring the scaling exponent of the integrated time series reveals the long-range correlation properties of the original series.

$$
y(i)=\sum_{j=1}^{i}[x(j)-\bar{x}] \quad i=1,2, \ldots ., N
$$

where $\mathrm{x}$ is the original time series and $\bar{x}$ is the mean of the series. The time domain is then divided into a $\mathrm{Ns}=\operatorname{int}(\mathrm{N} / \mathrm{s})$ distinct time segments of identical length $\mathrm{s}$. The length $\mathrm{N}$ of the time series may not be an integer multiple of the timescale s, and consequently a short portion at the end of the series may not be included in any segment. Therefore, the same process is performed from the opposite end of data, resulting in $2 \mathrm{Ns}$ time windows. A polynomial is fitted to the values of the series $\mathrm{y}$ in each segment, and then the variance for every segment is calculated as follows:

$$
\begin{aligned}
& F^{2}(s, v)=\frac{1}{s} \sum_{i=1}^{s}\left\{y[(v-1) s+i]-y_{t}(i)\right\}^{2} \quad v=1, \ldots, N_{s} \\
& F^{2}(s, v)=\frac{1}{S} \sum_{i=1}^{s}\left\{y\left[N-\left(v-N_{s}\right) s+i\right]-y_{t}(i)\right\}^{2} \quad v=N_{s+1}, \ldots, 2 N_{s}
\end{aligned}
$$

where $y_{t}(i)$ is the fitted polynomial in segment $v$, and the polynomial's degree remains constant for each segment. In the standard DFA, the fluctuation function is defined by the square root of the average of the variances over all segments.

$$
\mathrm{F}(\mathrm{s})=\left\{\frac{1}{2 \mathrm{~N}_{\mathrm{s}}} \sum_{\mathrm{v}=1}^{2 \mathrm{Ns}}\left[\mathrm{F}^{2}(\mathrm{~s}, \mathrm{v})\right]\right\}^{1 / 2}
$$

In the next step, the $\mathrm{q}^{\text {th }}$ order fluctuations function is determined by averaging the variances over all segments, defined as (Movahed \& Hermanis, 2008):

$$
\mathrm{F}_{\mathrm{q}}(\mathrm{s})=\left\{\frac{1}{2 \mathrm{~N}_{\mathrm{s}}} \sum_{\mathrm{v}=1}^{2 \mathrm{Ns}}\left[\mathrm{F}^{2}(\mathrm{~s}, \mathrm{v})\right]^{\mathrm{q} / 2}\right\}^{1 / \mathrm{q}}
$$


Finally, if the time series exhibits fractal properties, the scaling exponent $h(q)$, also called the generalized Hurst exponent, is obtained using the following power-law correlation (Kantelhardt et al., 2002; Q. Zhang et al., 2011).

$$
F_{q}(s) \alpha s^{h(q)}
$$

Of note, different orders of DFA, linear (DFA1), quadratic (DFA2), or higher-order polynomial, having different detrending capabilities, may be employed in the fitting process to eliminate trends in the time series (Kantelhardt, Koscielny-Bunde, Rego, Havlin, \& Bunde, 2001). Generally, river flow data have been described using DFA1 in the literature (Koscielny-Bunde et al., 2006).

Considering the stationary time series, $\mathrm{h}(\mathrm{q}=2)$ is identical to the well-known Hurst exponent. Likewise, the function $\mathrm{h}(\mathrm{q})$ is called the generalized Hurst exponent in literature (Kantelhardt et al., 2002). In these cases, the time series can be categorized as long-range correlated (persistent) if $h(2)>0.5$, or longrange uncorrelated (anti-persistent) if $h(2)<0.5$. Moreover, a value of $h(2)=0.5$ indicates uncorrelated series (Movahed \& Hermanis, 2008; X. Yu et al., 2016) . If the time series is multifractal, the scaling exponents are subject to variation in q. Otherwise, the scaling exponents are independent of various values of q. Note that when q values are positive, segments with large variance will govern the fluctuation function and the associated scaling exponents $\mathrm{h}(\mathrm{q})$ will describe the scaling properties of segments with large fluctuations. Correspondingly, negative values of $\mathrm{h}(\mathrm{q})$ describe the scaling behavior of segments with small fluctuations.

A customary way to address the multifractality in a time series is to employ the singularity spectrum, $\mathrm{f}(\alpha)$, according to Eq. 7 (Kantelhardt et al., 2002; Krzyszczak et al., 2017). The associated diagram is also called the Holder diagram.

$$
f(\alpha)=q[\alpha-h(q)]+1
$$

where $\alpha$ is the Holder exponent, defined as: 


$$
\alpha=\mathrm{h}(\mathrm{q})+\mathrm{q} \frac{\Delta(\mathrm{h}(\mathrm{q}))}{\Delta \mathrm{q}}
$$

The multifractal properties can be obtained from the shape of the Holder spectrum. For instance, the strength of multifractality can be taken as the width of the Holder diagram (Fan \& Lin, 2017; Kantelhardt et al., 2002). In addition to the generalized Hurst exponent, $\tau(\mathrm{q})$ is also considered as a scaling exponent, defined by Eq. 9. This exponent is entitled the mass exponent or the classical multifractal scaling exponent in the literature (Kantelhardt et al., 2002; Z.-G. Yu et al., 2014)

$$
\tau(\mathrm{q})=\mathrm{qh}(\mathrm{q})-1
$$

A common technique to elucidate the multifractal source is to evaluate the corresponding shuffled dataset using MF-DFA. In the process of shuffling, the time series are rearranged randomly, so that longrange correlations are demolished. If the multifractality stems from long-range correlation, the scaling exponents linked to the shuffled series $\left(h_{\text {shuf }}(q)\right)$ should be equal to 0.5 , regardless of the $q$ values. In the case of multifractality due to the broadness of the PDF, the scaling exponents are unchanged by the shuffling process.

\subsection{Extreme value removal}

To evaluate the influence of extreme values $(\mathrm{EV})$ on the fractal properties of the streamflow time series, extreme events were removed from the original time series using a published method (He \& Wang, 2017). The original time series $\mathrm{x}(\mathrm{i})$ was sorted in ascending order to obtain a new time series $\mathrm{z}(\mathrm{i})$ ( $\mathrm{i}=1$ to $\mathrm{N}$ ). Accordingly, the extremities are located at the beginning and end parts of $z(i)$. By removing the first and last $\mathrm{T} / 2$ data from $\mathrm{z}(\mathrm{i})$, an $\mathrm{EV}$ removed series $\mathrm{u}(\mathrm{i})(\mathrm{i}=1$ to $\mathrm{N}-\mathrm{T})$ was obtained where $\mathrm{T}$ is the number of extreme values, which was assumed to be $\mathrm{T}=0.05 \times \mathrm{N}$. The removed data were replaced randomly with non-extreme data points chosen from $\mathrm{u}(\mathrm{i})$ to form a series $\mathrm{w}(\mathrm{i})$ with $\mathrm{N}$ data point. Finally, the time series w(i) were rearranged to create EV-removed series with the same ranking as the original time series. 


\section{Results and discussion}

\subsection{Power spectra analysis of river discharge time series}

The power spectra of daily and hourly discharge data for the Shapur river, recorded from 2005 to 2016, are shown in Fig. 3 for both gauging stations. Decreasing trends are evident in the diagram, implying the presence of the scaling property. If straight lines are fitted to the log-log plots (Fig. 3), the slope of these lines indicates the spectral exponent, $\beta$ (see Eq. 1).

\subsection{Fractal properties of river discharge}

Overall three sets of discharge data were analyzed; i) daily data starting from 1978, ii) daily data starting from 2005, and iii) hourly data recording from 2005. The DFA analysis of all datasets revealed three scaling regions which represented two crossover times (point of changes in the scaling behavior), considering both daily and hourly records at the Chiti and Bushigan gauging stations (Fig. 4). Interestingly, the hourly and daily records exhibited similar fractal properties owing to identical crossover timescales and rather similar scaling exponents. The corresponding crossover times of these series are shown in Table 1. The first crossover was estimated to be between 69 and 83 days for different datasets, while the second was between 346 and 382 days, which may indicate seasonal and annual cycles in the Shapur river. The average values, i.e. 75 and 366 days, can be considered the first and second crossover times. The estimated scaling exponents of the daily and hourly discharge series were approximately equal, considering different timescales (Table 2). The relative difference between the Hurst exponents resulting from the daily and hourly signals was lower than $5 \%$, which shows that the daily records preserve the fractal properties of the hourly records. In addition, it appears that the scaling behavior at Chiti and Bushigan gauging stations is the same, which may be the result of a correlation between the flow rates at these two stations. It should be noted that Both stations are located on a single river, and no important tributary branch enters the river 
between the gauging stations. Thus, the possible fluctuations of both stations are similar.

For timescales lower than 75 days, the discharge time series mentioned above are persistent, because the scaling exponent ranged from 0.77 to 0.93 , i.e., it was greater than 0.5 . This indicates that a large value is more likely followed by a large value and a small value is probably followed by a small value (Kantelhardt, 2009). However, there was an anti-persistent correlation in long time periods (timescales longer than 366 days), for which the Hurst exponent was smaller than 0.5 , meaning that whenever the time series has been high in the past, it is more likely to be low in the future. Accordingly, the river discharge time series may be classified as fractional Gaussian noise process (fGn) (stationary time series) in the abovementioned timescales, because the Hurst exponent is lower than 1. However, the river flow series may be categorized as fractional Brownian motion process (fBm) (non-stationary) for timescales between 75 and 366 days, on account of the scaling exponent being higher than 1 (Table 2) (Rakhshandehroo \& Amiri, 2012). Of note, the application of fGn and fBm evolved in hydrology from the works of Hurst in 1965 and Mandelbrot and Van Ness in 1968 (Molz, Liu, \& Szulga, 1997).

In cases where the scaling exponent exceeds 1, the actual Hurst exponent is calculated as $\mathrm{h}(2)-1$ (Kantelhardt et al., 2002; Movahed \& Hermanis, 2008). Therefore, the corresponding Hurst exponent ranged in value from 0.06 to 0.24 for timescales between 75 and 366 days, signifying anti-persistent correlation. A situation with a scaling exponent larger than 1 has been reported previously by (Y. Zhang \& Ge, 2013), who found a scaling exponent of $h(2)=1.17$ for sea level fluctuations at timescales between 186 and 400 days. Similar fractal behavior has also been observed in groundwater level fluctuations (Rakhshandehroo \& Amiri, 2012; X. Yu et al., 2016). These results indicate that hourly and daily discharge signals may be considered stationary or non-stationary, depending on the range of timescale. 


\subsection{Multifractal properties of river discharge}

In order to determine whether the daily and hourly discharge time series exhibited monofractal or multifractal characteristics, the effects of different moments on the fluctuation function of the runoff data were examined (Fig. 5). For a specified timescale, a variety of q values ended up with different slopes (scaling exponents), which was obtained from the log-log plot of the fluctuation functions against timescales. Therefore, the values of scaling exponents (slopes) strongly depended on q values, indicating multifractal sources in the hourly and daily time series of the river runoff data. In addition, with regard to Fig. 5, the same trend for the fluctuation function was observed for daily and hourly datasets at the Bushigan and Chiti gauging stations. Moreover, the existence of the above-mentioned crossover times (75 days and 366 days) can also be seen in this diagram for all datasets. These results show that the daily and hourly discharge data have close multifractal trends. In addition, the variations in $h(q)$ on small timescales were much greater than the variations in $\mathrm{h}(\mathrm{q})$ on large timescales, indicating that the degree of multifractality was higher on small timescales for all discharge signals.

Considering datasets recorded from 2005 to 2016, the existence of multifractality was also evident in the plot of $h(q)$ as a function of $q$ (Fig. 6). The value of $h$ decreased continuously with a rise in the value of q for all hourly and daily discharge data, which signifies the existence of multifractal sources. Above all, the diagrams of $\mathrm{h}(\mathrm{q})$ depicted for hourly datasets were closely similar to the diagrams for daily runoff data, indicating that time aggregation from hourly resolution to daily resolution led to similar multifractal properties of streamflow data.

Moreover, small fluctuations (negative q) led to greater scaling exponents than large fluctuations (positive q), as frequently observed in multifractal time series (Kalamaras et al., 2017). However, the slope of the curves linked to the negative values of $q$ was steeper than the slope of the curves obtained from the positive $q$ values, confirming that small fluctuations may appear more often than large fluctuations in daily and hourly discharge signals. Of note, the segments of time series with small variances will govern the 
fluctuation function (Eq. 5) when $\mathrm{q}$ is negative. Hence, the $\mathrm{h}$ values obtained from negative values of $\mathrm{q}$ describe the scaling characteristics of the segments with small fluctuations. Considering positive q values, the segments with large variances will dominate the fluctuation function (Kantelhardt et al., 2002).

It can also be deduced that different scaling exponents are required to consider self-affinity in river discharge time series, whether recorded daily or hourly.

With reference to multifractal sources, the variations in $h_{\text {shuf }}(q)$ with respect to $q$ are also displayed in Fig. 6. As shown, the scaling exponent of the daily and hourly shuffled datasets was approximately equal to 0.5 , irrespective of the $\mathrm{q}$ value, indicating that the multifractal properties predominantly arise from different long-range correlations for small and large fluctuations.

The strength of multifractality of the discharge signals was measured using the singularity spectrum (Holder diagram). The singularity spectra of the daily and hourly discharge data corresponding to the Bushigan and the Chiti gauging stations are presented in Fig. 7. Since the variations of $h(q)$ versus $q$ experience decreasing tendency, its derivative $(\Delta(\mathrm{h}(\mathrm{q})) / \Delta \mathrm{q})$ is negative. Considering Eq. 8, the left of the singularity spectrum is associated with positive values of $\mathrm{q}$ and the right is linked to negative $\mathrm{q}$ values. In all cases, the diagrams have right tails and left truncations, indicating that these signals have multifractal structures that are more sensitive to local fluctuations with small magnitudes. The variations in $f(\alpha)$ on the right of the singularity spectrum are greater than the variations in $f(\alpha)$ on the left, indicating higher degrees of multifractality for negative values of q, which confirms the results in Fig. 6.

The multifractal characteristics of the above-mentioned signals are given in Table 3. The estimated value of $w$ (width of the singularity spectrum) for the daily and hourly discharge signals varied between 1.09 and 3.32, confirming the multifractal richness of the hourly and daily discharge signals. In line with Table 3, the hourly dataset led to slightly greater spectrum width than the daily dataset, denoting slightly higher multifractal richness in the hourly discharge signal. Consequently, averaging from hourly records to 
daily values will end up with only a small loss of information that appears negligible. In addition, the parameter $\Delta \mathrm{S}$, which measures quantitatively the shifting of the singularity spectrum, is defined as:

$$
\begin{aligned}
& \Delta \mathrm{S}=\mathrm{R}-\mathrm{L} \\
& \mathrm{R}=\alpha_{\text {max }}-\alpha_{0} \\
& \mathrm{~L}=\alpha_{0}-\alpha_{\text {min }}
\end{aligned}
$$

The value of $\Delta \mathrm{S}$ was positive for all signals (Table 3), confirming that the time series are more dependent upon small fluctuations than large-magnitude oscillations. Alternatively, the multifractal behavior of the time series mentioned above is perceived from the variations of $\tau(q)$ versus $q$ (Fig. 8). When a dataset is multifractal, the mass exponent is a nonlinear function of $\mathrm{q}$.

\subsection{Effect of extreme discharge values}

The MF-DFA approach was applied to the EV-removed series to examine the influence of extreme events on the multifractal properties. The changes in the scaling exponent $h(q)$ with $q$ for the EV-removed series are shown in Fig. 6. Similar to the original time series, there were decreasing trends in $\mathrm{h}$ versus q curves for all daily and hourly EV-removed signals, indicating that these datasets are multifractal. However, the variation in the scaling exponents resulting from EV-removed series was smaller than that in the original series. For instance, with an increase in q from -4 to 4 , h declined from 3.52 to 0.96 with the daily discharge signal at Chiti station, while h declined from 2.25 to 1.09 with the EV-removed daily signal at that station.

For negative values of $q$, the scaling exponents stemming from EV-removed series were smaller than the $h$ values obtained for the original series. In contrast, for positive q values the scaling exponents of EVremoved series were larger than those of the original data.

The corresponding singularity spectra of the EV-removed series are shown in Fig. 7. As can be seen, the width of singularity spectra associated with the EV-removed series declined in comparison with the original series in all cases, showing the contribution of extreme events to the degree of multifractality. The 
associated multifractal properties of the EV-removed series are given in Table 4. For the daily discharge time series at the Chiti station, the multifractality width dropped by approximately half in the EV-removed series, signifying that rare events may play a key role in the multifractality of streamflow signals. It should be highlighted that there was still noticeable multifractality in the EV-removed discharge signals, whether daily or hourly. Analogous to the original time series, the singularity spectra of EV-removed signals were right-skewed (Fig. 7). The decrease in the multifractality of EV removed series is also perceived from Fig. 8. The EV removed series experience flatter curves in comparison with the original time series, indicating smaller degrees of multifractality.

\subsection{Application of fractal analysis in flood prediction}

Historical streamflow data are mainly available in coarse time resolution (daily or monthly) (Torabi Haghighi \& Klöve., 2013; Rebora et al., 2016; Fazel et. al., 2017; Reynolds et al., 2017). Therefore, statistical temporal downscaling methods are widely employed to provide synthetic time series with finer time resolution (Rebora et al., 2016; Reynolds et al., 2017), which is critical for forecasting flood peaks. However, there is some uncertainty regarding the validity of time series with finer resolution generated using different downscaling methods. According to the present study, the scaling properties of hourly discharge data are approximately identical to the scaling properties of daily streamflow data. This property may be used in predicting hourly discharge time series from historical daily records, which is beneficial for determining peak flow rates in flood events. However, more time series of river streamflow with different time resolutions should be investigated to confirm this. In addition, it is possible to produce an artificial long discharge series, with scaling properties identical to those of present river flow data, in order to assist prediction of extreme floods. 


\section{Conclusions}

The scaling behavior of hourly and daily discharge time series of the Shapur river in Fars province, Iran, was measured using DFA, MF-DFA. Hourly and daily datasets from two gauging stations on the river were used to examine the influence of time aggregation on streamflow time series. In addition, the influences of rare events on the fractal behavior of the time series were studied.

The results of DFA indicated that the hourly and daily streamflow signals had similar scaling properties, so that the effect of time aggregation from hourly to daily resolution on the fractal properties of river runoff data was negligible. Discharge time series for the river might be classified as stationary series when the time scale was less than 75 days or greater than 366 days. For timescales between 75 and 366 days, both hourly and daily signals might exhibit non-stationary behavior.

In MF-DFA analyses, the scaling exponents of both discharge time series decreased with increasing q values, indicating the presence of multifractality. In shuffled time series, the main source of multifractality was different long-range correlations for small and large fluctuations. In addition, the singularity spectra resulting from both hourly and daily discharge data were left-truncated and right-skewed, indicating that multifractality is more sensitive to fine structure than local fluctuations with large magnitude.

When extreme events were omitted from the discharge signals, the width of the singularity spectra decreased. This indicates the contribution of extreme events to the multifractal behavior of both hourly and daily records and will permit more accurate estimation of extreme events in the future.

\section{DATA AVAILABILITY STATEMENT}

The data that support the findings of this study are available from the corresponding author upon reasonable request. 


\section{Acknowledgment}

The authors would like to thank the Fars regional water authority for permission of using the discharge data. The authors declare no conflict of interest.

\section{References}

Afshin, Y. (1994). The Iranian rivers. Tehran, Iran: Ministry of Power- Jamab engineering company.

Al Sawaf, M. B., Kawanisi, K., Kagami, J., Bahreinimotlagh, M., \& Danial, M. M. (2017). Scaling characteristics of mountainous river flow fluctuations determined using a shallow-water acoustic tomography system. Physica A: Statistical Mechanics and its Applications, 484, 11-20.

Ashraf F.B, Torabi Haghighi A, Marttila H \& Kløve B. Assessing impacts of climate change and river regulation on flow regimes in cold climate: A study of a pristine and a regulated river in the subarctic setting of Northern Europe. (2016), Journal of Hydrology, 542:410-22.

Ashraf F.B, Haghighi AT, Riml J, Alfredsen K, Koskela JJ, Kløve B \& Hannu Marttila. (2018). Changes in short term river flow regulation and hydropeaking in Nordic rivers. Scientific Reports, 8 (1).

Breslin, M. C., \& Belward, J. A. (1999). Fractal dimensions for rainfall time series. Mathematics and Computers in Simulation, 48(4), 437-446.

Cadenas, E., Campos-Amezcua, R., Rivera, W., Espinosa-Medina, M. A., Méndez-Gordillo, A. R., Rangel, E., \& Tena, J. (2019). Wind speed variability study based on the Hurst coefficient and fractal dimensional analysis. Energy Science \& Engineering, 7(2), 361-378.

Dutta, S., Ghosh, D., \& Chatterjee, S. (2018). Multifractal detrended cross correlation analysis of neurodegenerative diseases-An in depth study. Physica A: Statistical Mechanics and its Applications, 491, 188-198.

Fan, X., \& Lin, M. (2017). Multiscale multifractal detrended fluctuation analysis of earthquake magnitude series of Southern California. Physica A: Statistical Mechanics and its Applications, 479, 225-235.

Habib, A., Sorensen, J. P. R., Bloomfield, J. P., Muchan, K., Newell, A. J., \& Butler, A. P. (2017). Temporal scaling phenomena in groundwater-floodplain systems using robust detrended fluctuation analysis. Journal of hydrology, 549, 715-730.

He, S., \& Wang, Y. (2017). Revisiting the multifractality in stock returns and its modeling implications. Physica A: Statistical Mechanics and its Applications, 467, 11-20.

Hernández Ayala, J. J., Keellings, D., Waylen, P. R., \& Matyas, C. J. (2017). Extreme floods and their relationship with tropical cyclones in Puerto Rico. Hydrological Sciences Journal, 62(13), 21032119. 
Hoffmann, H., Baranowski, P., Krzyszczak, J., Zubik, M., Sławiński, C., Gaiser, T., \& Ewert, F. (2017). Temporal properties of spatially aggregated meteorological time series. Agricultural and forest meteorology, 234, 247-257.

Kalamaras, N., Philippopoulos, K., Deligiorgi, D., Tzanis, C., \& Karvounis, G. (2017). Multifractal scaling properties of daily air temperature time series. Chaos, Solitons \& Fractals, 98, 38-43.

Kantelhardt, J. W. (2009). Fractal and multifractal time series. Encyclopedia of Complexity and Systems Science, 3754-3779.

Kantelhardt, J. W., Koscielny-Bunde, E., Rego, H. H., Havlin, S., \& Bunde, A. (2001). Detecting longrange correlations with detrended fluctuation analysis. Physica A: Statistical Mechanics and its Applications, 295(3-4), 441-454.

Kantelhardt, J. W., Zschiegner, S. A., Koscielny-Bunde, E., Havlin, S., Bunde, A., \& Stanley, H. E. (2002). Multifractal detrended fluctuation analysis of nonstationary time series. Physica A: Statistical Mechanics and its Applications, 316(1), 87-114.

Koscielny-Bunde, E., Kantelhardt, J. W., Braun, P., Bunde, A., \& Havlin, S. (2006). Long-term persistence and multifractality of river runoff records: Detrended fluctuation studies. Journal of hydrology, 322(1), 120-137.

Krzyszczak, J., Baranowski, P., Zubik, M., \& Hoffmann, H. (2017). Temporal scale influence on multifractal properties of agro-meteorological time series. Agricultural and Forest Meteorology, 239, 223-235.

Kuai, K. Z., \& Tsai, C. W. (2012). Identification of varying time scales in sediment transport using the Hilbert-Huang Transform method. Journal of hydrology, 420, 245-254.

Iran meteorological Organization (2018). http://www.irimo.ir/far/wd/2703.

Livina, V., Ashkenazy, Y., Kizner, Z., Strygin, V., Bunde, A., \& Havlin, S. (2003). A stochastic model of river discharge fluctuations. Physica A: Statistical Mechanics and its Applications, 330(1), 283290.

Malamud, B. D., \& Turcotte, D. L. (1999). Self-affine time series: measures of weak and strong persistence. Journal of statistical planning and inference, 80(1-2), 173-196.

Maskey, M. L., Puente, C. E., \& Sivakumar, B. (2019). Temporal downscaling rainfall and streamflow records through a deterministic fractal geometric approach. Journal of hydrology, 568, 447-461.

Molz, F., Liu, H., \& Szulga, J. (1997). Fractional Brownian motion and fractional Gaussian noise in subsurface hydrology: A review, presentation of fundamental properties, and extensions. Water Resources Research, 33(10), 2273-2286.

Movahed, M. S., \& Hermanis, E. (2008). Fractal analysis of river flow fluctuations. Physica A: Statistical Mechanics and its Applications, 387(4), 915-932. 
Mustonen, k., Mykrä, H., Marttila, H., Torabi Haghighi, A., Kløve, B., Aroviita, J.; Veijalainen, N., Sippel, K., Muotka, T. (2019). Defining natural flow regimes of highly seasonal boreal rivers: relationship with taxonomical and functional structure of macroinvertebrate communities. Freshwater Science, 35.

Peng, C.-K., Buldyrev, S., Havlin, S., Simons, M., Stanley, H., \& Goldberger, A. (1994). Mosaic organization of DNA nucleotides (Vol. 49).

Peng, C. K., Havlin, S., Stanley, H. E., \& Goldberger, A. L. (1995). Quantification of scaling exponents and crossover phenomena in nonstationary heartbeat time series. Chaos: An Interdisciplinary Journal of Nonlinear Science, 5(1), 82-87.

Piskorski, J., Kosmider, M., Mieszkowski, D., Krauze, T., Wykretowicz, A., \& Guzik, P. (2018). Properties of Asymmetric Detrended Fluctuation Analysis in the time series of RR intervals. Physica A: Statistical Mechanics and its Applications, 491, 347-360.

Rakhshandehroo, G. R., \& Amiri, S. M. (2012). Evaluating fractal behavior in groundwater level fluctuations time series. Journal of hydrology, 464, 550-556.

Rebora, N., Silvestro, F., Rudari, R., Herold, C., \& Ferraris, L. (2016). Downscaling stream flow time series from monthly to daily scales using an auto-regressive stochastic algorithm: StreamFARM. Journal of hydrology, 537, 297-310.

Rego, C., Frota, H., \& Gusmão, M. (2013). Multifractality of Brazilian rivers. Journal of hydrology, 495, 208-215.

Rehman, S. (2009). Study of Saudi Arabian climatic conditions using Hurst exponent and climatic predictability index. Chaos, Solitons \& Fractals, 39(2), 499-509.

Reynolds, J., Halldin, S., Xu, C.-Y., Seibert, J., \& Kauffeldt, A. (2017). Sub-daily runoff predictions using parameters calibrated on the basis of data with a daily temporal resolution. Journal of hydrology, $550,399-411$.

Rizvi, S. A. R., \& Arshad, S. (2016). How does crisis affect efficiency? An empirical study of East Asian markets. Borsa Istanbul Review, 16(1), 1-8.

Ronchail, J., Espinoza, J. C., Drapeau, G., Sabot, M., Cochonneau, G., \& Schor, T. (2018). The flood recession period in Western Amazonia and its variability during the 1985-2015 period. Journal of Hydrology: Regional Studies, 15, 16-30.

Saha, D., Shaw, P. K., Ghosh, S., Janaki, M. S., \& Sekar Iyengar, A. N. (2018). Quantification of scaling exponent with Crossover type phenomena for different types of forcing in DC glow discharge plasma. Physica A: Statistical Mechanics and its Applications, 490, 300-310.

Salas, J., Obeysekera, J., \& Vogel, R. (2018). Techniques for assessing water infrastructure for nonstationary extreme events: a review. Hydrological Sciences Journal, 63(3), 325-352. 
Tawn, J., Shooter, R., Towe, R., \& Lamb, R. (2018). Modelling spatial extreme events with environmental applications. Spatial Statistics.

Teng, Y., \& Shang, P. (2018). Detrended fluctuation analysis based on higher-order moments of financial time series. Physica A: Statistical Mechanics and its Applications, 490, 311-322.

Torabi Haghighi A, Marttila H \& Kløve B. (2014). Development of a new index to assess river regime impacts after dam construction. Global and Planetery Change,122, 186-96.

Torabi Haghighi A, Bin Ashraf F, Riml J, Koskela J, Kløve B, Marttila H. (2019), A power market-based operation support model for sub-daily hydropower regulation practices. Applied Energy, 255.

Yu, X., Ghasemizadeh, R., Padilla, I. Y., Kaeli, D., \& Alshawabkeh, A. (2016). Patterns of temporal scaling of groundwater level fluctuation. Journal of hydrology, 536, 485-495.

Yu, Z.-G., Leung, Y., Chen, Y. D., Zhang, Q., Anh, V., \& Zhou, Y. (2014). Multifractal analyses of daily rainfall time series in Pearl River basin of China. Physica A: Statistical Mechanics and its Applications, 405, 193-202.

Zhang, Q., Zhou, Y., Singh, V. P., \& Chen, Y. D. (2011). Comparison of detrending methods for fluctuation analysis in hydrology. Journal of hydrology, 400(1), 121-132.

Zhang, Y., \& Ge, E. (2013). Temporal scaling behavior of sea-level change in Hong Kong-Multifractal temporally weighted detrended fluctuation analysis. Global and Planetary Change, 100, 362-370. 


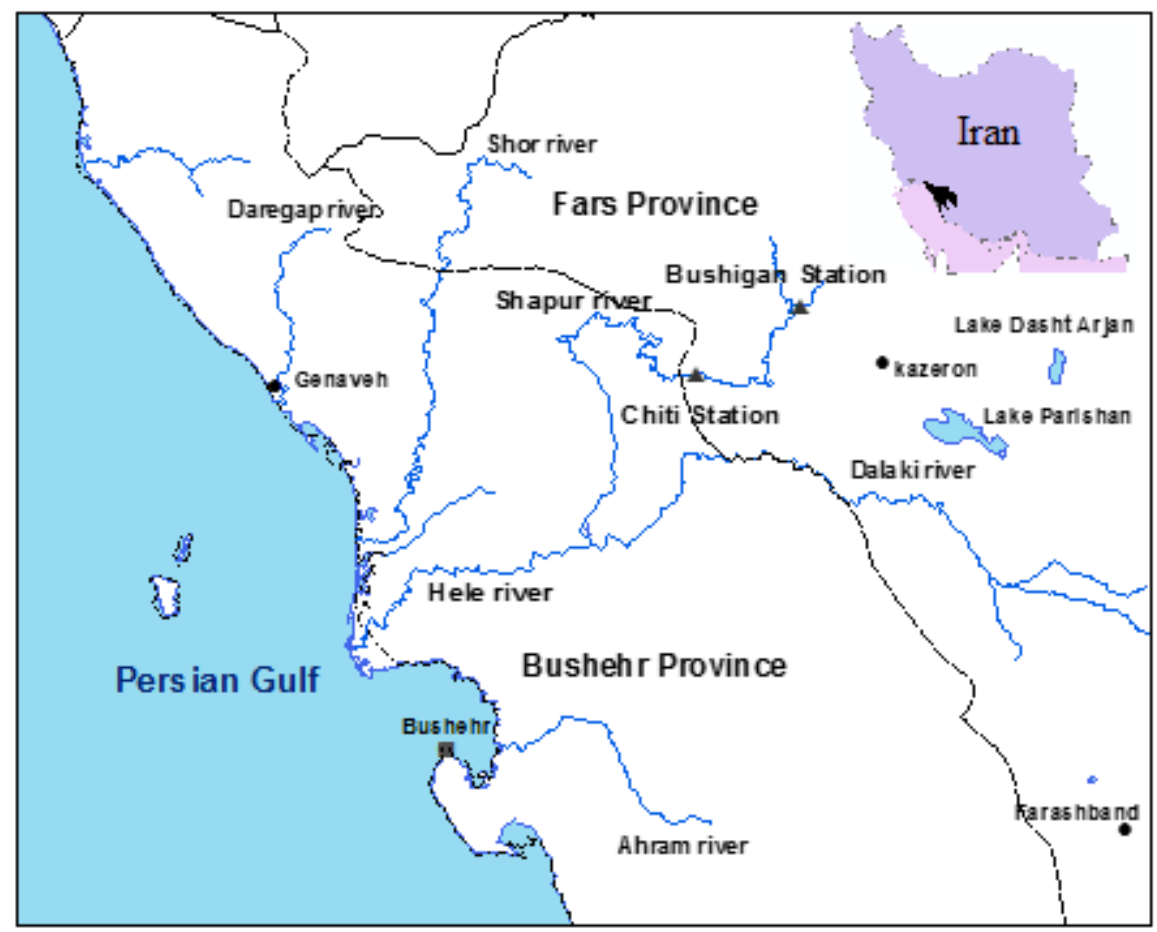

Fig. 1. Heleh basin in Fars province, Iran, and location of the Bushigan and Chiti gauging stations on the Shapur river. 

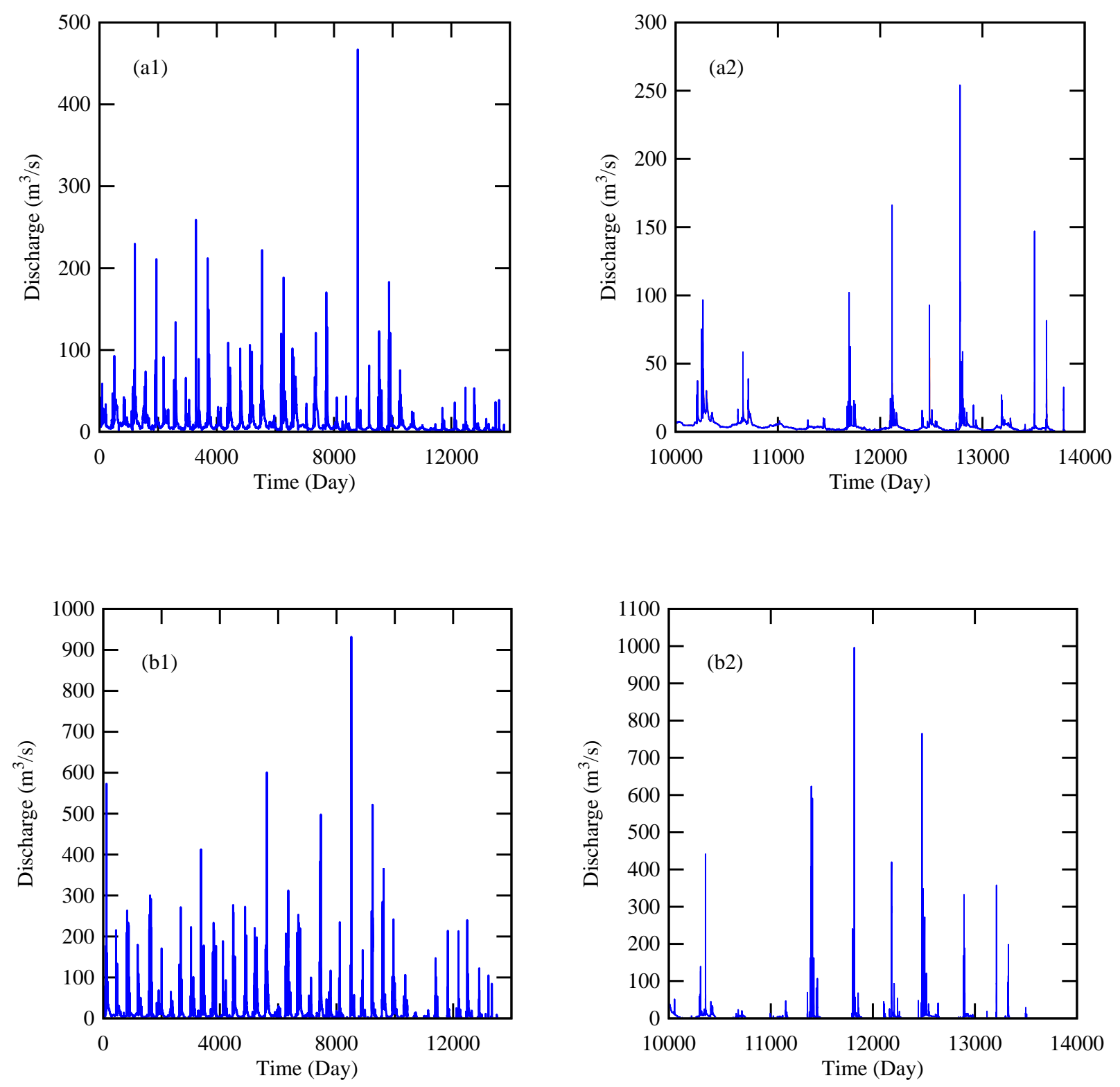

Fig. 2. Discharge time series of the Shapur river at (a) Bushigan and (b) Chiti gauging station. 1) Daily dataset (1978-2016), 2) hourly dataset (2005-2016). 

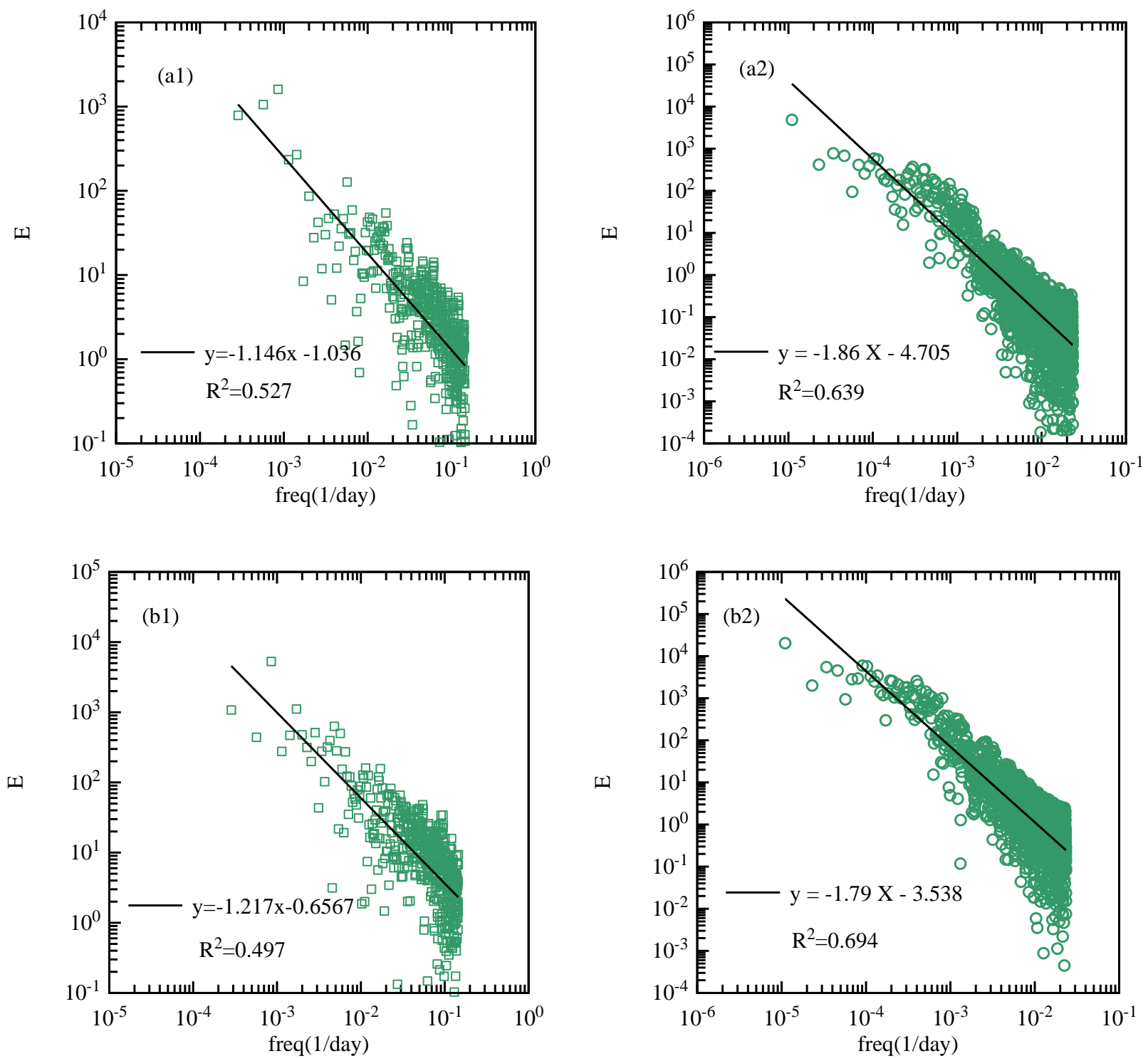

Fig. 3. Log-log power spectrum of the daily discharge time series at (a) Bushigan and (b) Chiti gauging station. 1) Daily dataset (2005-2016), 2) hourly dataset (2005-2016). 


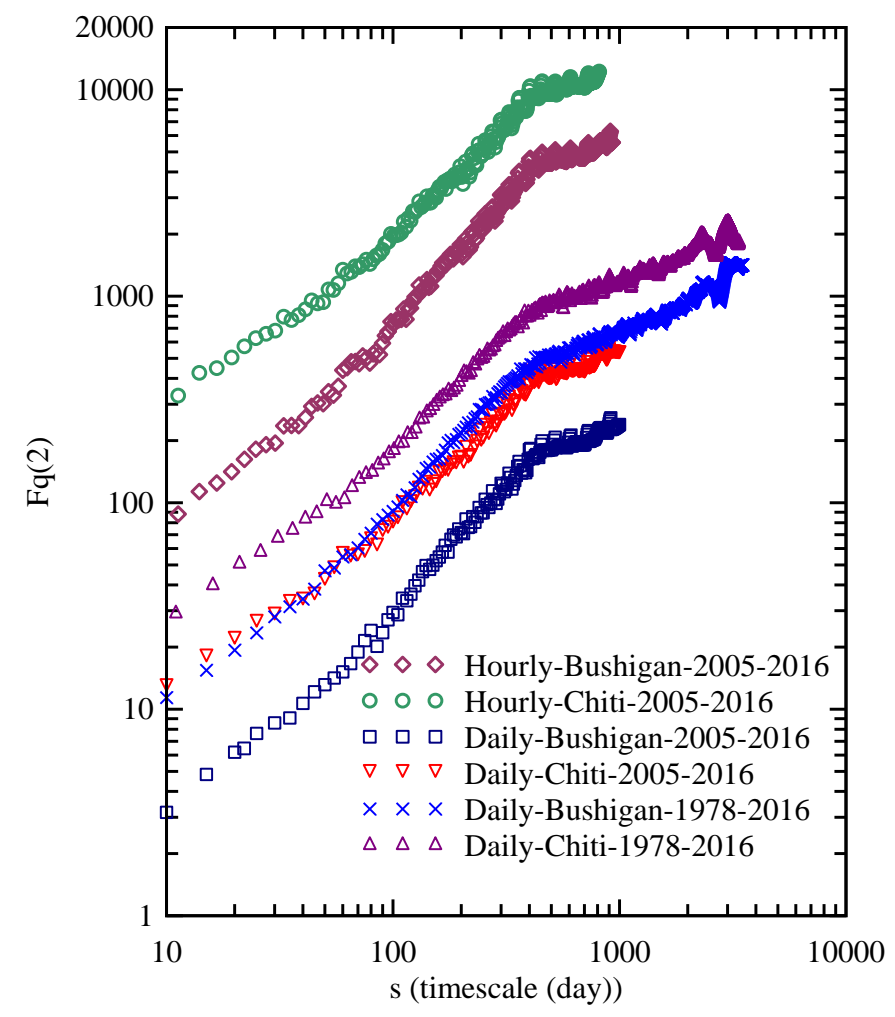

Fig. 4. Results of Detrended Fluctuation Analysis (DFA) on daily and hourly discharge time series at Bushigan and Chiti gauging station. 

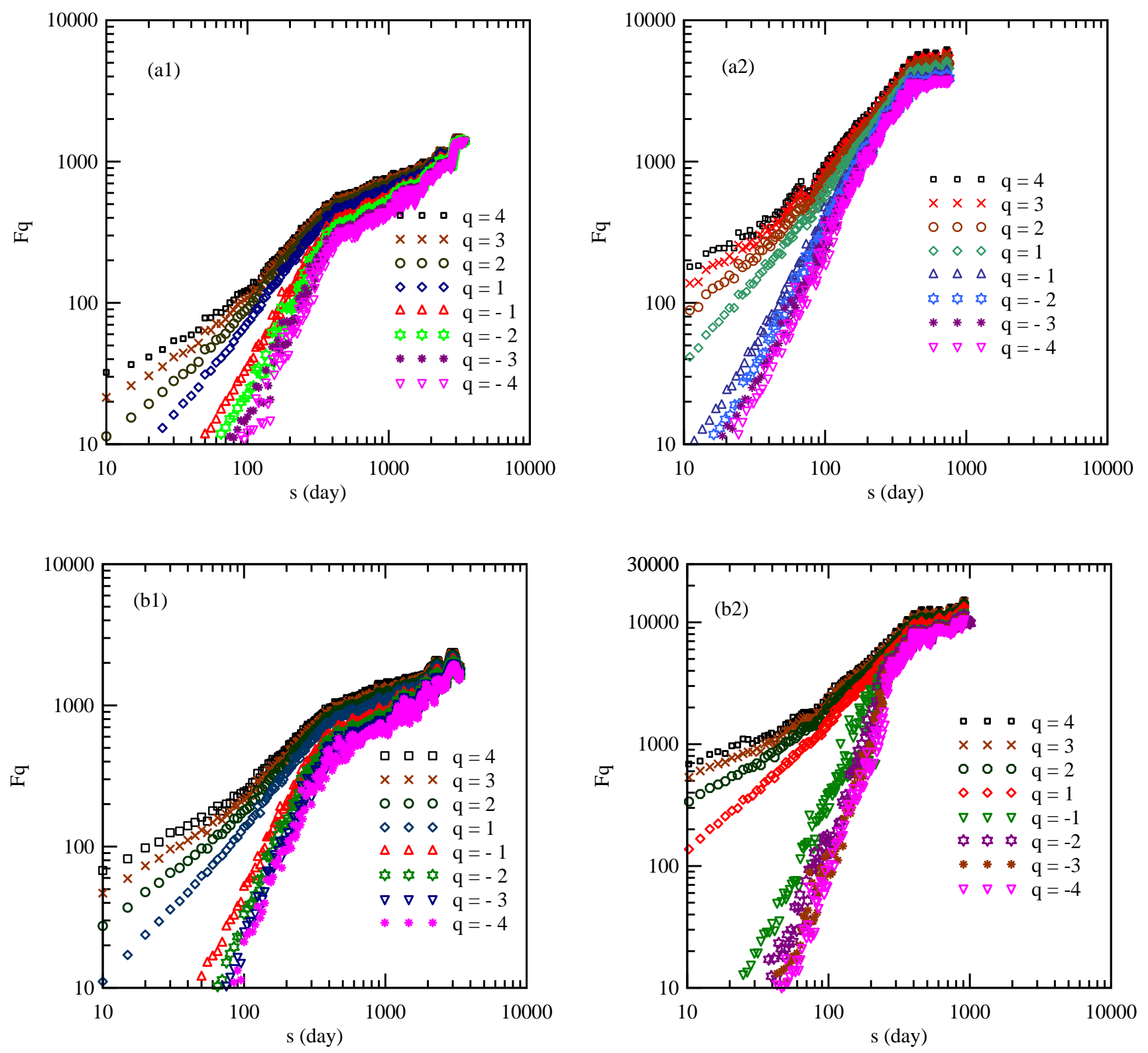

Fig. 5. Fluctuation function $F_{q}$ versus timescale $s$ for the discharge time series at (a) Bushigan and (b) Chiti gauging station. 1) Daily dataset (1978-2016), 2) hourly dataset (2005-2016). 

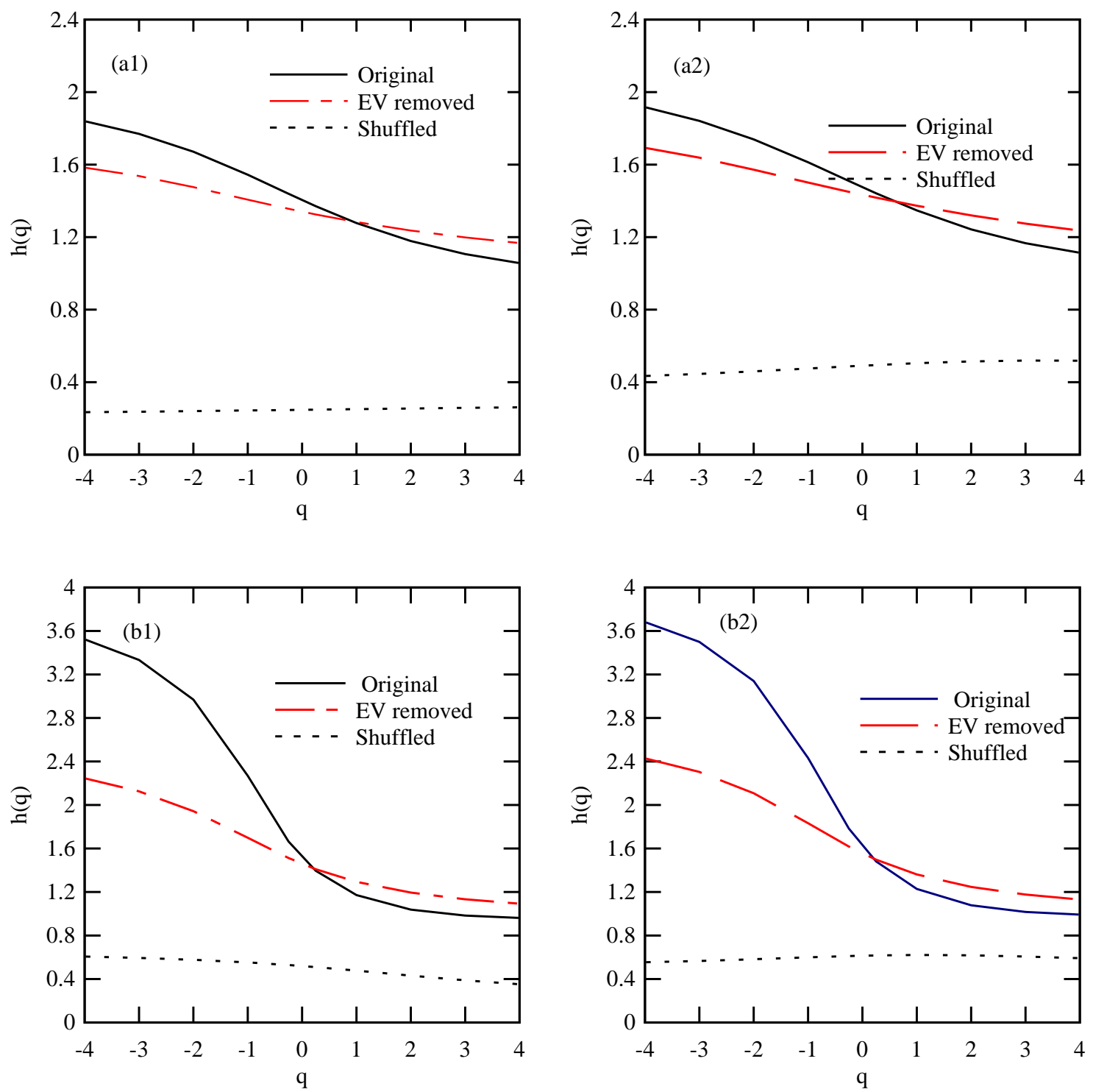

Fig. 6. Scaling exponent $h(q)$ versus $q$ for the discharge time series at (a) Bushigan and (b) Chiti gauging station. 1) Daily dataset (2005-2016), 2) hourly dataset (2005-2016). 

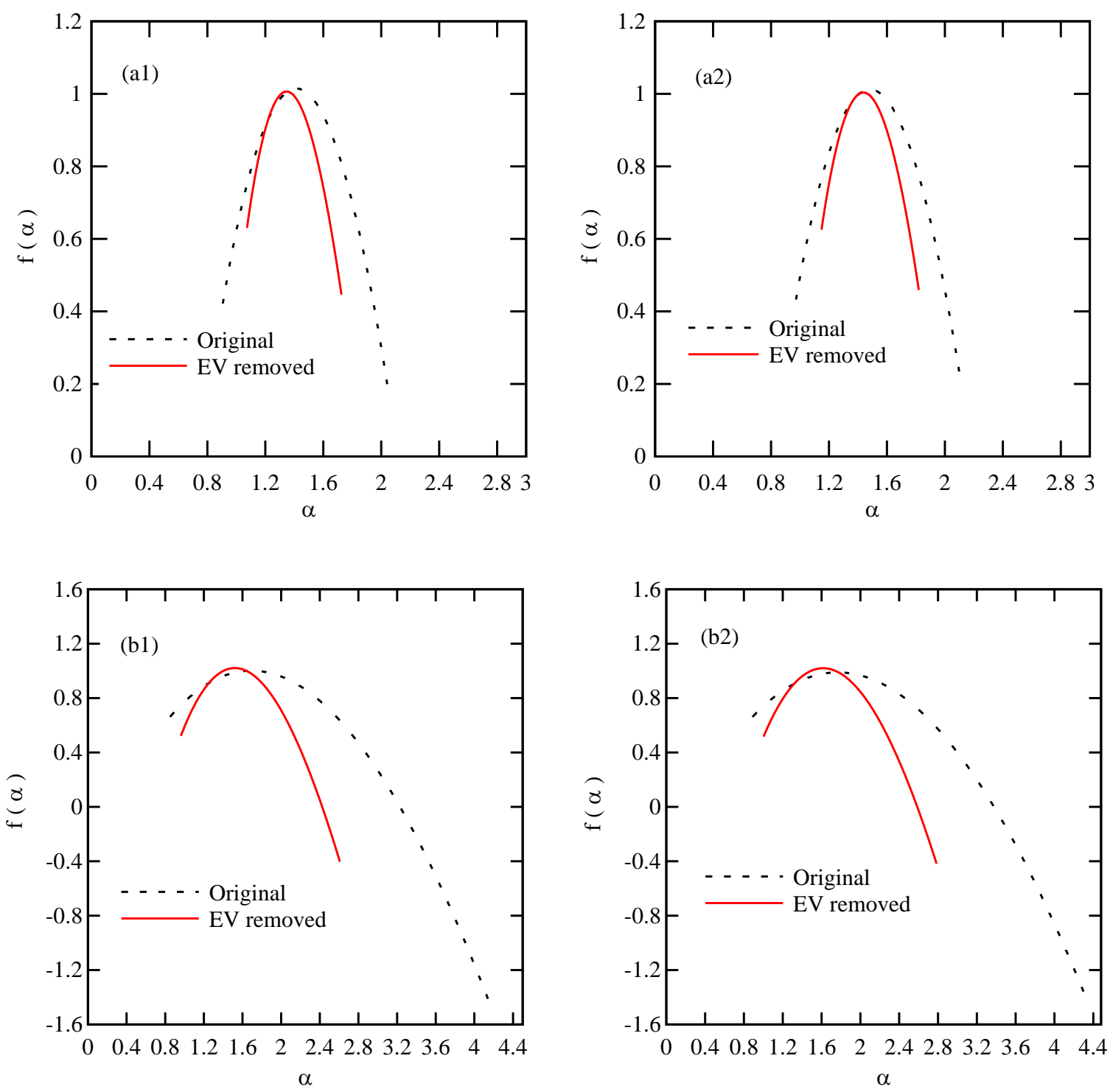

Fig. 7. Singularity spectrum for original discharge time series and times series with extreme values (EV) removed at (a) Bushigan and (b) Chiti gauging station. 1) Daily dataset (2005-2016), 2) hourly dataset (2005-2016). 

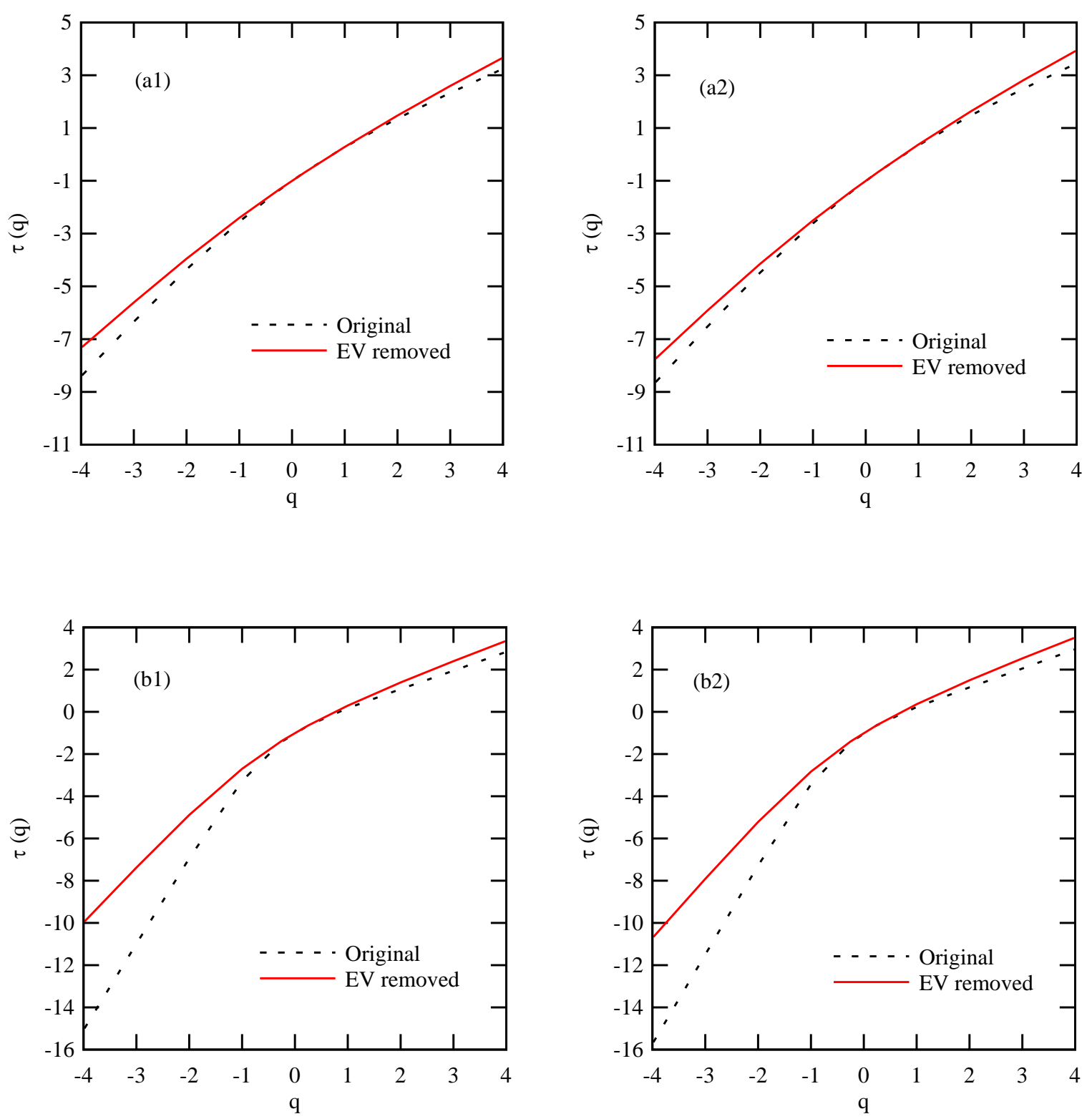

Fig. 8. Mass exponent $\mathrm{h}(\mathrm{q})$ versus $\mathrm{q}$ for the discharge time series at (a) Bushigan and (b) Chiti gauging station. 1) Daily dataset (2005-2016), 2) hourly dataset (2005-2016). 
